\title{
A controllability result for the 1-D isentropic Euler equation
}

\author{
Olivier Glass *
}

\section{Introduction}

\subsection{The equations}

The problem that we consider concerns the one-dimensional isentropic Euler equations, which is a classical model of gas dynamics:

$$
\left\{\begin{array}{l}
\partial_{t} \rho+\partial_{x}(m)=0, \\
\partial_{t}(m)+\partial_{x}\left(\frac{m^{2}}{\rho}+\kappa \rho^{\gamma}\right)=0 .
\end{array}\right.
$$

In the above equation, $t$ is the time $x$ is the position, $\rho=\rho(t, x) \geq 0$ is the density of the fluid, $m(t, x)$ is the momentum $\left(v(t, x)=\frac{m(t, x)}{\rho(t, x)}\right.$ is the velocity of the fluid), the pressure law is $p(\rho)=\kappa \rho^{\gamma}, \gamma \in(1,3]$.

Equation (EI) is formulated in Eulerian coordinates. The problem of one-dimensional isentropic gas dynamics is also frequently studied in Lagrangian coordinates:

$$
\left\{\begin{array}{l}
\partial_{t} \tau-\partial_{x} v=0 \\
\partial_{t} v+\partial_{x}\left(\kappa \tau^{-\gamma}\right)=0
\end{array}\right.
$$

where here $\tau:=1 / \rho$ is the specific volume. System $(\mathrm{P})$ is commonly referred as the $p$-system.

For classical solutions as well as for some classes of weak entropy equations, both systems are known to be equivalent (see [28]) via a suitable change of coordinates, when both problems are considered on the spatial domain $\mathbb{R}$. Here the problems that we will consider are posed in the interval $[0,1]$, and consequently the controllability problems that we will describe below are different whether we consider system (EI) or system $(\mathrm{P})$.

\subsection{The controllability problem}

The problem under consideration is the following. We consider equations $(\mathrm{EI})$ and $(\mathrm{P})$ for $(t, x) \in[0, T] \times[0,1]$, but we do not provide boundary conditions on $[0, T] \times\{0,1\}$, since here boundary conditions will be considered as a control, that is, a way to influence the system that we have at our disposal. We define the state of the system at time $t$ as the whole function $u=(\rho, m)$ (resp. $u=(\tau, v))$ on $[0,1]$ in the case (EI) (resp. (P)). Now the question is the following: given two possible states of the system, say $u_{0}$ and $u_{1}$, can we choose the control suitably, in order that the solution of the system starting from $u_{0}$, eventually reaches $u_{1}$ at time $T$ ?

Let us underline that the boundary conditions for such hyperbolic systems of conservation laws are in general quite involved (see for instance [14]). To overcome this difficulty, we reformulate the controllability problem in the following underdetermined form: given $u_{0}, u_{1}$ and $T$, can we find a solution of (EI) or (P) (which are underdetermined systems since no boundary conditions is imposed) such that

$$
u_{\mid t=0}=u_{0} \text { and } u_{\mid t=T}=u_{1} ?
$$

\footnotetext{
${ }^{*}$ Laboratoire Jacques-Louis Lions, Université Pierre et Marie Curie, Boîte courrier 187, 75252 Paris Cedex 05, France.
} 


\subsection{Class of solutions}

It is a classical statement that systems (EI) and (P) are hyperbolic systems of conservation laws, which in general develop singularities in finite time. It is hence natural to consider discontinuous (weak) solutions. As well-known, such weak solutions are no longer unique, and it is natural to consider weak solutions which satisfy entropy conditions aimed at singling out the physically relevant solution. These entropy conditions are the following:

Definition 1 We define an entropy/entropy flux couple as a couple of functions $(\eta, q)$ such that

$$
\forall u \in \mathbb{R}^{+*} \times \mathbb{R}, \quad D \eta(u) \cdot D f(u)=D q(u) .
$$

Then entropy solutions are defined as weak solutions of the system,

$$
u_{t}+(f(u))_{x}=0
$$

which moreover satisfy that, for all $(\eta, q)$ entropy couple with $\eta$ convex, stands, in the sense of distributions:

$$
\eta(u)_{t}+q(u)_{x} \leq 0
$$

Now to be more specific, the solutions that we will consider here are $B V$ entropy solutions: precisely, the solutions will have small total variation (in $x$ ) uniformly in time, and they avoid vacuum. The method that we will use to construct solutions of the above problem is the front-tracking algorithm. Let us emphasize that the class of solutions that we consider is not, by far, the most general class of solutions in which existence theory is established. Indeed, the following general result stands:

Theorem 1 (Lions, Perthame, Souganidis, [23]) Let $\left(\rho_{0}, v_{0}\right) \in L^{\infty}(\mathbb{R}), \rho_{0} \geq 0$. Then for all $\gamma>1$, there exists a global entropy solution of $(E I)$ with initial data $\left(\rho_{0}, v_{0}\right)$.

\section{Basic facts on systems of conservation laws}

Before giving the precise results discussed here, let us recall some basic material concerning hyperbolic systems of conservation laws in general, and systems (EI) and (P) in particular.

\subsection{Basic notations}

Systems (EI) and (P) can be written the following general form of hyperbolic systems of conservations laws, that is:

$$
u_{t}+f(u)_{x}=0
$$

where $f: \mathbb{R}^{n} \rightarrow \mathbb{R}^{n}$ is a smooth function called the flux. The Jacobian matrix $A(u):=d f(u)$ satisfies the strict hyperbolicity conditions, viz.

$$
A(u):=d f(u) \text { has } n \text { real distinct eigenvalues } \lambda_{1}<\cdots<\lambda_{n} \text {, }
$$

with corresponding eigenvectors $r_{i}(u)$.

To be more specific, these eigenvalues are given by

- Case (EI): $u=(\rho, m)$ :

$$
\lambda_{1}=\frac{m}{\rho}-\sqrt{\kappa \gamma} \rho^{\frac{\gamma-1}{2}} \text { and } \lambda_{2}=\frac{m}{\rho}+\sqrt{\kappa \gamma} \rho^{\frac{\gamma-1}{2}},
$$

- Case $(\mathrm{P}): u=(\tau, v)$ :

$$
\lambda_{1}=-\sqrt{\kappa \gamma \tau^{-\gamma-1}} \text { and } \lambda_{2}=\sqrt{\kappa \gamma \tau^{-\gamma-1}}
$$


Hence systems (EI) and (P) satisfy the strict hyperbolicity condition as long as $0<\rho<+\infty$.

Moreover, both systems (EI) and (P) satisfy the condition of genuine non-linearity in the sense of Lax: for both $i=1$ and 2 , the fields $\left(\lambda_{i}, r_{i}\right)$ satisfy:

$$
\nabla \lambda_{i}(u) . r_{i}(u) \neq 0 \quad \text { for all } u .
$$

As a consequence, in the sequel, we normalize $r_{i}$ in order that $\nabla \lambda_{i} \cdot r_{i}=1$.

Let us recall the celebrated result of Glimm [17], that when the system satisfies the strict hyperbolicity conditions, and has all its characteristic fields genuinely nonlinear in the sense of Lax, then if the initial condition $u_{0} \in B V(\mathbb{R})$ has small enough total variation, then there is a global entropy solution of the system starting from $u_{0}$.

Let us also recall that both systems can be endowed with coordinates for the states which are composed of Riemann invariants. We recall that $w^{i}$ is a $i$-Riemann invariant if it satisfies $r_{i}$. $\nabla w^{i}=0$. Pairs of Riemann invariants for systems (EI) and (P) are given by

- Case (EI):

$$
w^{1}(u)=\frac{m}{\rho}+\frac{2 \sqrt{\kappa \gamma}}{\gamma-1} \rho^{\frac{\gamma-1}{2}} \text { and } w^{2}(u)=\frac{m}{\rho}-\frac{2 \sqrt{\kappa \gamma}}{\gamma-1} \rho^{\frac{\gamma-1}{2}},
$$

- Case $(\mathrm{P})$ :

$$
w^{1}(u)=v+\frac{2 \sqrt{\kappa \gamma}}{\gamma-1} \tau^{-\frac{\gamma-1}{2}} \text { and } w^{2}(u)=v-\frac{2 \sqrt{\kappa \gamma}}{\gamma-1} \tau^{-\frac{\gamma-1}{2}} .
$$

The rest of this section intends to recall shortly the principle of the construction of a solution by means of the front-tracking algorithm (we restrict our attention to the $2 \times 2$ systems which is what we need in the present work).

\subsection{The Riemann problem and the front-tracking algorithm}

The Riemann problem. The front-tracking algorithm is based upon the so-called Riemann problem, that is, the following one: find an autosimilar solution $u=\bar{u}(x / t)$ to the initial-value problem:

$$
\left\{\begin{array}{l}
u_{t}+(f(u))_{x}=0 \text { in } \mathbb{R}^{+} \times \mathbb{R}, \\
u_{|\{0\} \times| \mathbb{R}^{-}}=u_{l} \text { and } u_{\mid\{0\} \times \mathbb{R}^{+}}=u_{r} \text { in } \mathbb{R},
\end{array}\right.
$$

where $u_{l}$ and $u_{r}$ are constant states. The solution of this problem is very classical (see Lax [20]), and is obtained by introducing Lax's wave curves, which consist of points that can be joined starting from $u_{l}$ either by a $i$-shock or a $i$-rarefaction wave, as described below.

We begin with the shock waves: these are discontinuities satisfying Rankine-Hugoniot relations and Lax's inequalities:

$$
\begin{gathered}
f\left(u_{r}\right)-f\left(u_{l}\right)=s\left[u_{r}-u_{l}\right], \\
\lambda_{i}\left(u_{r}\right)<s<\lambda_{i}\left(u_{l}\right) \\
\lambda_{i-1}\left(u_{l}\right)<s<\lambda_{i+1}\left(u_{r}\right) .
\end{gathered}
$$

where $s$ is the speed of the shock, which gives the particular solution

$$
u(t, x)=\left\{\begin{array}{l}
u_{l} \text { for } x / t<s \\
u_{r} \text { for } x / t>s .
\end{array}\right.
$$


Then the rarefaction waves are defined by introducing integral curves of $r_{i}$ :

$$
\left\{\begin{array}{l}
\frac{d}{d \sigma} R_{i}(\sigma)=r_{i}\left(R_{i}(\sigma)\right), \\
R_{i}(0)=u_{l}, \\
\sigma \geq 0
\end{array}\right.
$$

The state $u_{r}=R_{i}(\bar{\sigma}), \bar{\sigma} \geq 0$ can be joined to $u_{l}$ via a rarefaction wave which is given by

$$
u(t, x)=\left\{\begin{array}{l}
u_{l} \text { for } x / t<\lambda_{i}\left(u_{l}\right) \\
R_{i}(\sigma) \text { for } x / t=\lambda_{i}\left(R_{i}(\sigma)\right), \\
u_{r} \text { for } x / t>\lambda_{i}\left(u_{r}\right) .
\end{array}\right.
$$

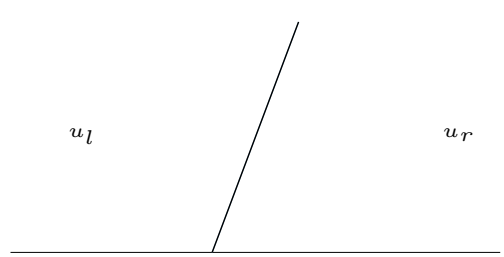

(a) Shock $\left(u_{l}, u_{r}\right)$

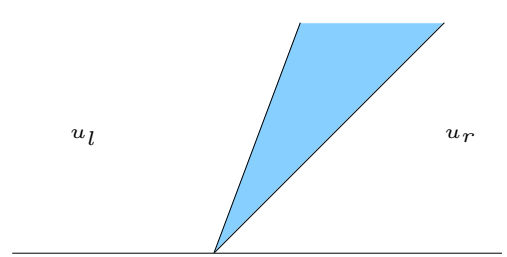

(b) Rarefaction wave $\left(u_{l}, u_{r}\right)$

Figure 1: Simple waves

Both wave curves (corresponding to each characteristic family) are known to be regular. We give in figure 2 a representation of these wave curves for system (EI), in $(\rho, m)$ and Riemann coordinates. Critical curves are curves where one of the characteristic speeds vanishes.

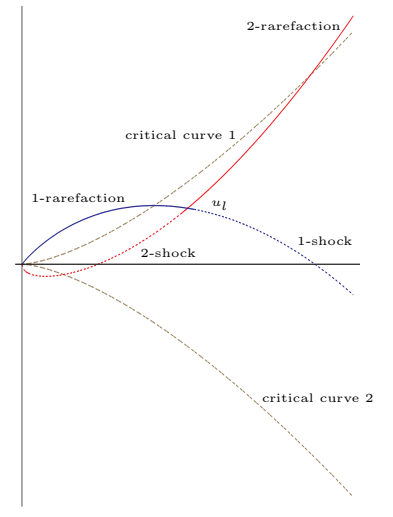

(a) $(\rho, m)$ coordinates

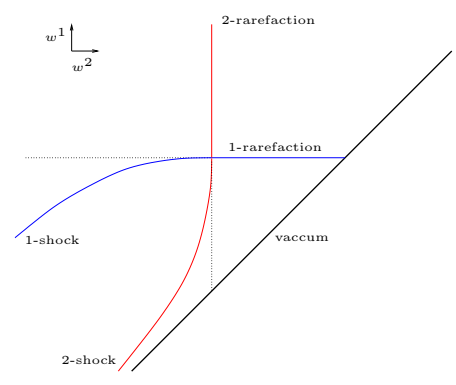

(b) $\left(w^{1}, w^{2}\right)$ coordinates

Figure 2: Wave curves

Then Lax's Theorem proves that one can solve (at least locally) the Riemann problem by first following the 1-curve then the 2-curve, such as described in the figure 3.

Front-tracking algorithm. Let us now shortly describe the construction by front-tracking algorithm. This method was introduced by Dafermos [12] in the framework of scalar equations, then extended by Di Perna [13] to $2 \times 2$ genuinely nonlinear systems (see also Alber [1]), and then extended to $n \times n$ systems by Bressan [6], Risebro [25], ... 


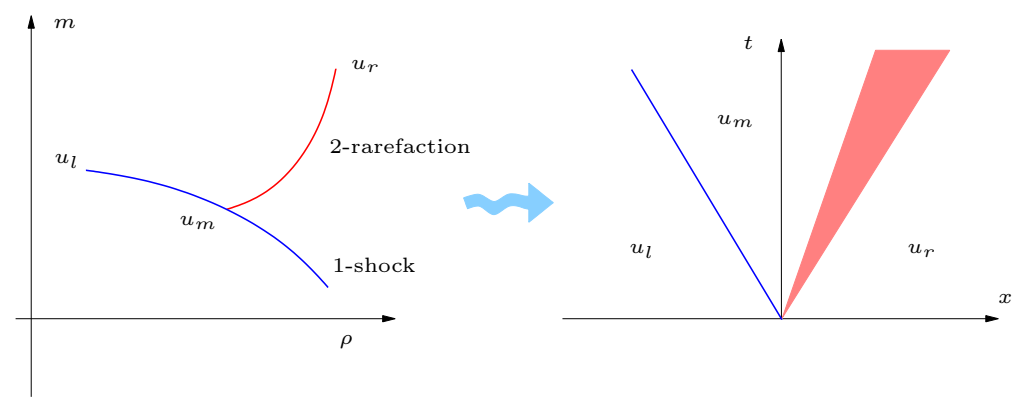

Figure 3: Solution of the Riemann problem

We briefly describe the construction in the case of $2 \times 2$ systems. The idea is to construct a sequence of piecewise constant approximations of a solution. To construct these approximations, the algorithm is the following:

- Approximate initial condition by piecewise constant functions:

$$
T V\left(u^{n}\right) \leq T V\left(u_{0}\right) \text { and }\left\|u^{n}-u_{0}\right\|_{L^{1}} \rightarrow 0 \text { as } n \rightarrow+\infty .
$$

- Solve the Riemann problems at each discontinuity points of $u^{n}$,

- Replace the rarefaction waves by rarefaction fans (see below).

- At this step, we have constructed for small times a piecewise constant approximation, in which constant states are separated by straight lines called fronts. These are either shock fronts or rarefaction fronts. Points at which these fronts cross are called interaction points.

- to extend the approximation over interaction points, iterate the procedure without splitting again rarefaction fronts.

This algorithm is represented in figure 4 .

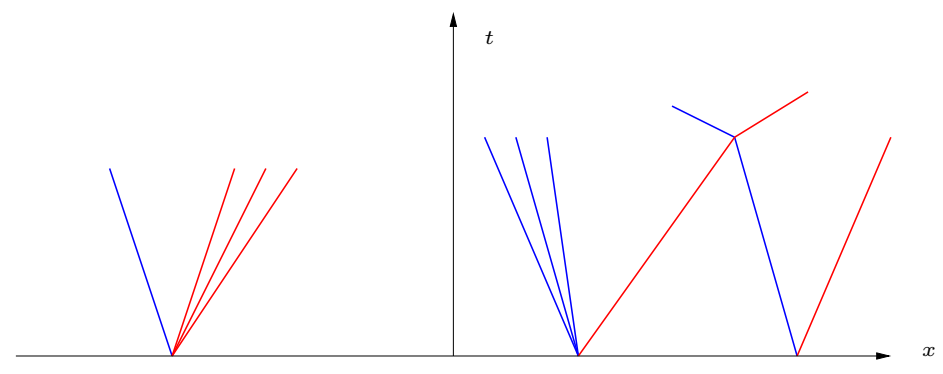

Figure 4: Front-tracking approximations

Replacing rarefaction waves with rarefaction fans means replacing the wave given by

$$
u(t, x)=\left\{\begin{array}{l}
u_{l} \text { for }\left(x-x_{0}\right) /\left(t-t_{0}\right)<\lambda_{i}\left(u_{l}\right) \\
R_{i}(\sigma) \text { for }\left(x-x_{0}\right) /\left(t-t_{0}\right)=\lambda_{i}\left(R_{i}(\sigma)\right), \\
u_{r} \text { for }\left(x-x_{0}\right) /\left(t-t_{0}\right)>\lambda_{i}\left(u_{r}\right) .
\end{array}\right.
$$


by

$$
u(t, x)=\left\{\begin{array}{l}
u_{l} \text { for }\left(x-x_{0}\right) /\left(t-t_{0}\right)<\lambda_{i}\left(u_{l}\right) \\
R_{i}\left(\sigma_{k}\right) \text { for } \lambda_{i}\left(R_{i}\left(\sigma_{k-1}\right)\right) \leq\left(x-x_{0}\right) /\left(t-t_{0}\right)<\lambda_{i}\left(R_{i}\left(\sigma_{k}\right)\right), k=1 \ldots K, \\
u_{r} \text { for }\left(x-x_{0}\right) /\left(t-t_{0}\right)>\lambda_{i}\left(u_{r}\right),
\end{array}\right.
$$

with $\sigma_{k}=k \bar{\sigma} / K$ and $K$ is a number chosen for instance in order that $\bar{\sigma} / K \leq \varepsilon$ where $\varepsilon$ is a threshold to be determined.

Then one shows than this defines a piecewise constant function, with a finite number of fronts and discrete interaction points. A central argument is due to Glimm: consider

$$
V(\tau)=\sum_{\alpha \text { wave at time } t}\left|\sigma_{\alpha}\right| ; \quad Q(\tau)=\sum_{\substack{\alpha, \beta \\ \text { approaching waves }}}\left|\sigma_{\alpha}\right| \cdot\left|\sigma_{\beta}\right|,
$$

Analyzing interactions $\alpha+\beta \rightarrow \alpha^{\prime}+\beta^{\prime}$ one shows that: for some $C>0$, if $T V\left(u_{0}\right)$ is small enough, then $V(t)+C Q(t)$ is non-increasing.

This allows to prove a uniform in time $B V$ estimate on the approximations, then a $\operatorname{Lip}_{t}\left(L_{x}^{1}\right)$ estimate by using the bounded speed of propagation. This gives compactness, and one shows that we get a suitable entropy solution in the limit. For more precisions on this algorithm, we refer to [1], [6] and [13].

\section{$3 \quad$ Results and previous works}

\subsection{Results}

Let us describe the results that we discuss here. First, concerning the system in Eulerian coordinates, we have:

Theorem 2 (Eulerian system) Consider $\bar{u}_{0}$ and $\bar{u}_{1}$ two states in $\mathbb{R}^{+*} \times \mathbb{R}$. Set $\overline{\lambda_{1}}:=\lambda_{1}\left(\bar{u}_{1}\right)$ and $\overline{\lambda_{2}}:=$ $\lambda_{1}\left(\bar{u}_{2}\right)$. For any $\alpha \in(0,1)$, there exist $\varepsilon_{1}=\varepsilon_{1}\left(\bar{u}_{0}\right)>0, \varepsilon_{2}=\varepsilon_{2}\left(\bar{u}_{1}, \alpha\right)>0$, and and $T=T\left(\bar{u}_{0}, \bar{u}_{1}\right)>0$, such that, for any $u_{0}, u_{1} \in B V([0,1])$ satisfying:

$$
\begin{aligned}
& \left\|u_{0}-\bar{u}_{0}\right\| \leq \varepsilon_{1} \text { and } T V\left(u_{0}\right) \leq \varepsilon_{1}, \\
& \left\|u_{1}-\bar{u}_{1}\right\| \leq \varepsilon_{2} \text { and } T V\left(u_{1}\right) \leq \varepsilon_{2},
\end{aligned}
$$

and $\forall x, y \in[0,1]$ such that $x<y$,

$$
\begin{aligned}
& \frac{w^{2}\left(u_{1}(x)\right)-w^{2}\left(u_{1}(y)\right)}{x-y} \leq c_{\gamma}(1-\alpha) \max \left(\frac{\overline{\lambda_{2}}-\overline{\lambda_{1}}}{1-y}, \frac{\overline{\lambda_{1}}}{x}, \frac{-\overline{\lambda_{1}}}{1-y}\right), \\
& \frac{w^{1}\left(u_{1}(x)\right)-w^{1}\left(u_{1}(y)\right)}{x-y} \leq c_{\gamma}(1-\alpha) \max \left(\frac{\overline{\lambda_{2}}-\overline{\lambda_{1}}}{x}, \frac{-\overline{\lambda_{2}}}{1-y}, \frac{\overline{\lambda_{2}}}{x}\right),
\end{aligned}
$$

there is an entropy solution $u$ of $(E I)$ in $[0, T] \times[0,1]$ such that

$$
u_{\mid t=0}=u_{0}, \text { and } u_{\mid t=T}=u_{1} .
$$

For the Lagrangian system, we have the following:

Theorem 3 (Lagrangian system) Consider $\bar{u}_{0}$ and $\bar{u}_{1}$ two states in $\mathbb{R}^{+*} \times \mathbb{R}$. Set $\bar{\lambda}_{1}:=\lambda_{1}\left(\bar{u}_{1}\right)$ and $\overline{\lambda_{2}}:=\lambda_{1}\left(\bar{u}_{2}\right)$. For any $\alpha \in(0,1)$, there exist $\varepsilon_{1}=\varepsilon_{1}\left(\bar{u}_{0}\right)>0, \varepsilon_{2}=\varepsilon_{2}\left(\bar{u}_{1}, \alpha\right)>0$, and and $T=T\left(\bar{u}_{0}, \bar{u}_{1}\right)>0$, such that, for any $u_{0}, u_{1} \in B V([0,1])$ satisfying:

$$
\begin{aligned}
&\left\|u_{0}-\bar{u}_{0}\right\| \leq \varepsilon_{1} \text { and } T V\left(u_{0}\right) \leq \varepsilon_{1}, \\
&\left\|u_{1}-\bar{u}_{1}\right\| \leq \varepsilon_{2} \text { and } T V\left(u_{1}\right) \leq \varepsilon_{2},
\end{aligned}
$$


and $\forall x, y \in[0,1]$ such that $x<y$,

$$
\begin{aligned}
& \frac{w^{2}\left(u_{1}(x)\right)-w^{2}\left(u_{1}(y)\right)}{x-y} \leq c\left(\gamma, \overline{u_{1}}\right)(1-\alpha) \frac{\overline{\lambda_{2}}-\overline{\lambda_{1}}}{1-y}, \\
& \frac{w^{1}\left(u_{1}(x)\right)-w^{1}\left(u_{1}(y)\right)}{x-y} \leq c\left(\gamma, \overline{u_{1}}\right)(1-\alpha) \frac{\overline{\lambda_{2}}-\overline{\lambda_{1}}}{x},
\end{aligned}
$$

there is an entropy solution $u$ of $(P)$ in $[0, T] \times[0,1]$ such that

$$
u_{\mid t=0}=u_{0}, \text { and } u_{\mid t=T}=u_{1} .
$$

Remark 1 Conditions (1)-(2) and (3)-(4) are close to Oleinik's estimates on genuinely nonlinear conservation laws. An important remark is that these conditions are not satisfied by the trajectories of systems (EI) or $(P)$ in general, such as described in figure 5. The interaction of two shocks of the same family gen-

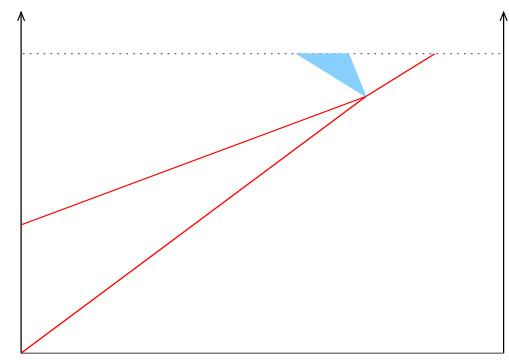

Figure 5: Non-necessity of the above conditions

erates a rarefaction wave in the other family; if this takes place shortly before $T$, then the above Oleinik-type inequalities are not satisfied.

\subsection{Previous works}

Before sketching the proofs of Theorems 2 and 3, let us describe some results that have been obtained in the field.

Classical solutions. There are several results concerning the control of hyperbolic systems (in particular for the system (EI) in the case $\gamma=2$ which corresponds to the Saint-Venant/Shallow water equation). We cite the following general result which concerns even systems in nonconservative form:

Theorem 4 (Li-Rao, [22], 2002) Consider

$$
\partial_{t} u+A(u) u_{x}=F(u),
$$

such that $A(u)$ has $n$ distinct real eigenvalues $\lambda_{1}(u)<\cdots<\lambda_{k}(u) \leq-c<0$ and $0 \leq c<\lambda_{k+1}(u)<\cdots<$ $\lambda_{n}(u)$. Then for all $\phi, \psi \in C^{1}([0,1])$ such that $\|\phi\|_{C^{1}}+\|\psi\|_{C^{1}}<\varepsilon$, there exists a solution $u \in C^{1}([0, T] \times[0,1])$ such that

$$
u_{\mid t=0}=\phi \text {, and } u_{\mid t=T}=\psi \text {. }
$$

Weak entropy solutions. We list below several results that concern controllability problems for hyperbolic systems of conservation laws, in the framework of entropy solutions. 
- Ancona and Marson [4] (1998): for the scalar equation $u_{t}+(f(u))_{x}=0$ with $f^{\prime \prime} \geq c>0$, they give a complete description of the attainable set starting from 0 .

- Horsin [19] (1998) has studied the controllability problem for the Burgers equation $u_{t}+\left(u^{2} / 2\right)_{x}=0$ with general initial data $u_{0} \in B V$ using Coron's return method, which is also a key ingredient in the present work.

- Bressan and Coclite [7] (2002): for systems with genuinely nonlinear fields and satisfying $\lambda_{1}(\cdot)<$ $\cdots<\lambda_{k}(\cdot) \leq-c<0$ and $0<c \leq \lambda_{k+1}(\cdot)<\cdots<\lambda_{n}(\cdot)$, for any constant state $\omega$, one can find $u$ such that

$$
u(t, \cdot) \rightarrow \omega \text { as } t \rightarrow+\infty .
$$

- Bressan and Coclite [7] (2002): for a class of systems containing Di Perna's system:

$$
\left\{\begin{array}{l}
\partial_{t} \rho+\partial_{x}(\rho u)=0, \\
\partial_{t} u+\partial_{x}\left(\frac{u^{2}}{2}+\frac{K^{2}}{\gamma-1} \rho^{\gamma-1}\right)=0,
\end{array}\right.
$$

there are initial conditions $\varphi \in B V([0,1])$ of arbitrary small total variation such that any entropy solution $u$ remaining of small total variation satisfies:

for any $t, u(t, \cdot)$ is not constant.

- Ancona and Coclite [3] (2002): Temple systems satisfying $\lambda_{1}(u)<\cdots<\lambda_{k}(u) \leq-c<0$ and $0<c \leq \lambda_{k+1}(u)<\cdots<\lambda_{n}(u)$, are controllable in $L^{\infty}$ provided the final state satisfies the Oleinik-type condition:

$$
\begin{aligned}
\frac{w^{i}\left(u_{1}(x)\right)-w^{i}\left(u_{1}(y)\right)}{x-y} \leq \frac{C}{1-y}, \text { for } i \leq k, \\
\frac{w^{i}\left(u_{1}(x)\right)-w^{i}\left(u_{1}(y)\right)}{x-y} \leq \frac{C}{x}, \text { for } i \geq k+1 .
\end{aligned}
$$

Essentially, the above condition is necessary, which contrasts with here (see Remark 1).

\section{Sketch of the proof in the Eulerian case}

Let us give the main ideas of the proof in the Eulerian case. The Lagrangian case which has several differences is discussed further.

The proof relies on Coron's return method: the idea is to connect $u_{0}$ and $u_{1}$ via a solution which which goes far away from $u_{0}$ and $u_{1}$. (Usually, the method states to find a particular solution, with identical initial and final states, around which the linearized system is controllable; here we prefer not to use this terminology since the linearization is delicate at this level of regularity.) This method was introduced in the context of a finite-dimensional control problem [9], and then used in several PDE problems, see for instance $[5,10,11,15,16,19]$.

The proof is split in three steps:

- Drive $u_{0}$ to a constant state,

- Drive the previous state to any constant state,

- Drive a constant state to $u_{1}$.

We describe the above steps in separate paragraphs. 


\subsection{First step: treating the initial condition}

Considering Bressan and Coclite's negative result that we cited above, it seems natural - even if the systems considered here do not belong to the class considered in [7] — not to consider a solution that stays close to $u_{0}$ and $u_{1}$, but rather a solution which is a strong perturbation with respect to initial and final states.

Hence, the idea is to make a strong 2 -shock enter the domain through the left side.

To that purpose, one considers $U_{l}$ a state connected to $\bar{u}_{0}$ (a constant state close to $u_{0}$ ) via a (strong) 2-shock:

$$
\left[\overline{\rho_{0}},+\infty\right) \ni \rho_{l} \mapsto\left(\rho_{l}, m_{l}\right) \text { with } \frac{m_{l}}{\rho_{l}}=\frac{\overline{m_{0}}}{\overline{\rho_{0}}}+\sqrt{\kappa \frac{1}{\rho_{l} \overline{\rho_{0}}} \frac{\rho_{l}^{\gamma}-\overline{\rho_{0}}}{\rho_{l}-\overline{\rho_{0}}}}\left(\rho_{l}-\overline{\rho_{0}}\right) .
$$

We choose $U_{l}$ suitably: we ask that both the shock speed of $\left(U_{l}, \bar{u}_{0}\right)$ and the first characteristic speed at $U_{l}$ are larger than 2. (As a consequence the second characteristic speed at $U_{l}$ will also be larger than 2.) This is possible because of these simple computations valid on the left shock curve:

$$
\begin{aligned}
& s=\frac{\overline{m_{0}}}{\overline{\rho_{0}}}+\sqrt{\kappa \frac{\rho_{l}}{\overline{\rho_{0}}} \frac{\rho_{l}^{\gamma}-\overline{\rho_{0}} \gamma}{\rho_{l}-\overline{\rho_{0}}}}, \\
& \lambda_{1}\left(\rho_{l}, m_{l}\right)=\frac{\overline{m_{0}}}{\overline{\rho_{0}}}+\sqrt{\kappa \frac{\rho_{l}}{\overline{\rho_{0}}} \frac{\rho_{l}^{\gamma}-\overline{\rho_{0}} \gamma}{\rho_{l}-\overline{\rho_{0}}}}\left(1-\frac{\overline{\rho_{0}}}{\rho_{l}}\right)-\sqrt{\kappa \gamma} \rho_{l}^{\frac{\gamma-1}{2}} .
\end{aligned}
$$

Now that we have chosen $U_{l}$, one constructs a solution on $\mathbb{R}$ with initial condition:

$$
\left\{\begin{array}{l}
U_{l} \text { on }(-\infty, 0), \\
u_{0} \text { on }[0,1] \\
\overline{u_{0}} \text { on }(1,+\infty)
\end{array}\right.
$$

Several authors (Alber [1], Schochet [27], Corli \& Sablé-Tougeron [8],...) have studied the existence of $B V$ solutions in the neighborhood of a strong shock, under Majda's stability condition [24]:

$$
\begin{aligned}
& \text { i. } \quad s \text { is not an eigenvalue of } A\left(u^{ \pm}\right), \\
& \text {ii. } \quad\left\{r_{j}\left(u^{+}\right) / \lambda_{j}\left(u^{+}\right)>s\right\} \cup\left\{u^{+}-u^{-}\right\} \cup\left\{r_{j}\left(u^{-}\right) / \lambda_{j}\left(u^{-}\right)<s\right\} \\
& \quad \text { is a basis of } \mathbb{R}^{2},
\end{aligned}
$$

which is satisfied for any shock here. Moreover, Schochet [27] proved that the Riemann problem is solvable in a neighborhood of the strong shock and gave interaction estimates on the interactions $\Gamma+\gamma \rightarrow \Gamma^{\prime}+\gamma^{\prime}$.

Using Schochet's interaction estimates, one can construct a global solution with the initial condition described above (this can be done approximately as described in Section 2).

Then, the central argument that makes the solution reach a constant state, is that in the front-tracking approximations, on the left of the 2-strong shock, all characteristic speeds are positive and bounded away from 0 . As a consequence, fronts leave the domain, such as described in figure 6 , which allows to conclude.

This yields a solution of the first step.

\subsection{Second step: travelling between constant states}

This step is very simple. In the states domain, there are three different zones:

$$
\begin{aligned}
& \mathcal{D}_{1}:=\left\{u, 0<\lambda_{1}<\lambda_{2}\right\}, \\
& \mathcal{D}_{2}:=\left\{u, \lambda_{1}<0<\lambda_{2}\right\}, \\
& \mathcal{D}_{3}:=\left\{u, \lambda_{1}<\lambda_{2}<0\right\} .
\end{aligned}
$$

The first point is to observe that inside each zone, one can move along (right or left) wave curves, using simple waves, such as described in the following figure:

Then it remains to observe that one move from a zone to another one by using strong shocks such as described here: 


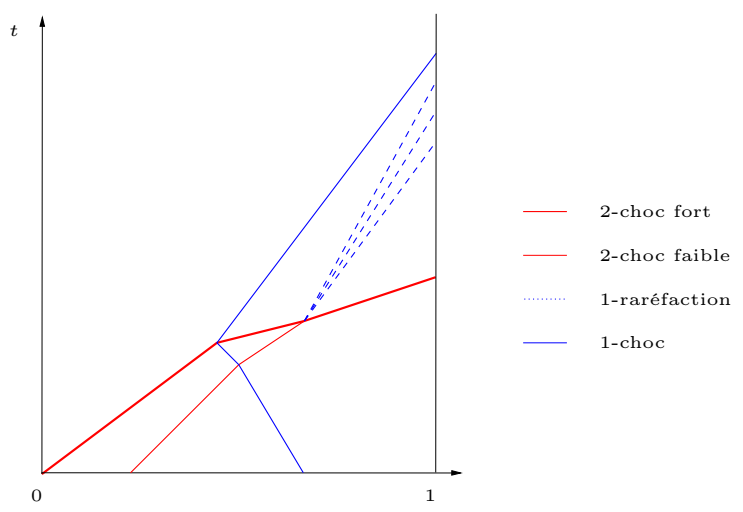

Figure 6: First step
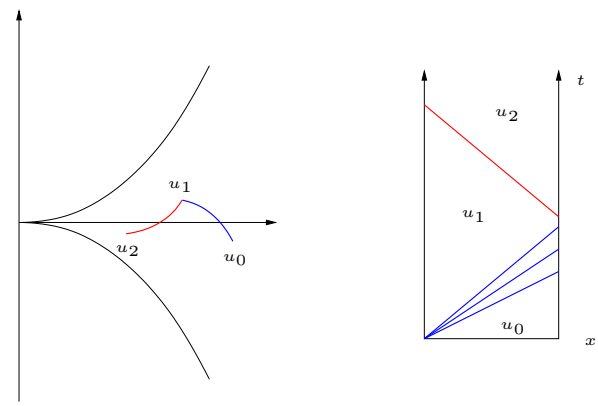

Figure 7: Travelling inside a zone
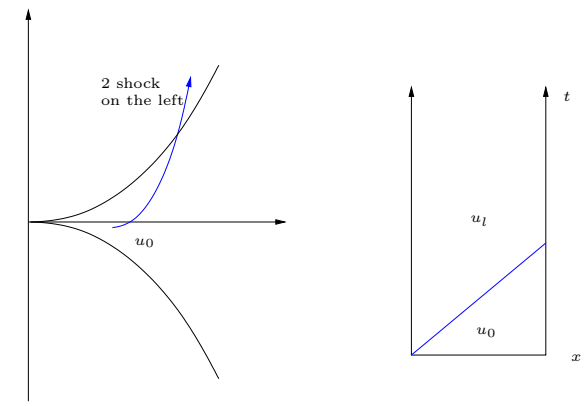

Figure 8: Moving between zones

\subsection{Third step: reaching the final state}

The idea is to construct a solution by a backward front-tracking algorithm, "starting" from the final state. To that purpose, it seems natural to solve the backward Riemann problem.

The difficulty comes from the fact that the backward Riemann problem is very ill-posed:

- Existence: Typically, two states which would generate a rarefaction wave in the usual motion of time, cannot be extended backward in a way that respects entropy criterions.

- Uniqueness: Even when a solution exists, there is no uniqueness in general. This is illustrated in 
the figure below, where $u_{l}$ and $u_{r}$ can be connected either by a single (backward) 2-shock or by two 2 -shocks then by a 1 -shock.
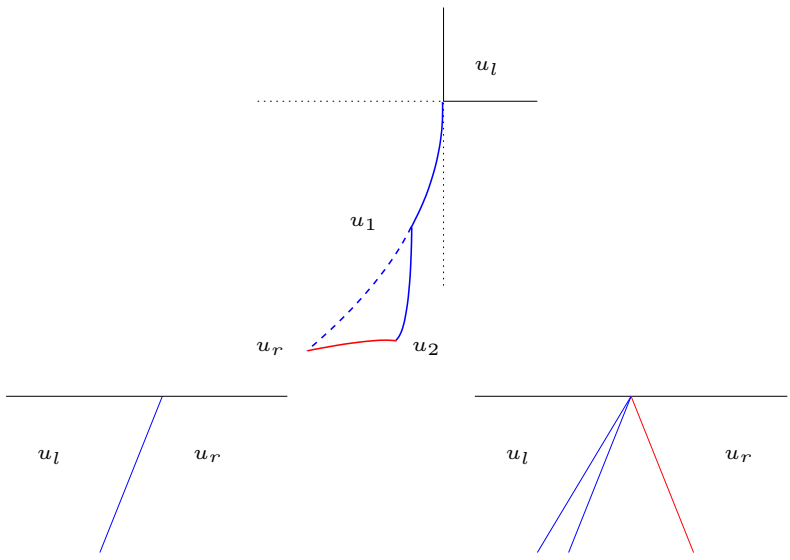

Figure 9: Non-uniqueness of the backward Riemann problem

We should bear in mind that, while the lack of existence of a solution to the backward Riemann problem is a serious issue (this is the main reason why we impose Oleinik-type inequalities in Theorems 2 and 3), on the contrary, the lack of uniqueness is clearly good news. Indeed, in most controllability problems, one does not expect the control solving the problem to be unique (unless adding supplementary expectations). Hence, not only this lack of uniqueness is not disappointing, but even this is advantageous, because this means that during the implementation of the backward front-tracking algorithm, we have some kind of a choice. This choice will be used later.

Another problem comes when trying to implement a backward front-tracking algorithm: the fact that characteristic speeds can be close to 0 . This is clearly an issue since fronts with null speed do not leave the domain, and we want to reach a constant state.

Now both problems get better when we make use of strong shocks. The idea is to construct a solution which includes strong shocks such as described in figure 10 .
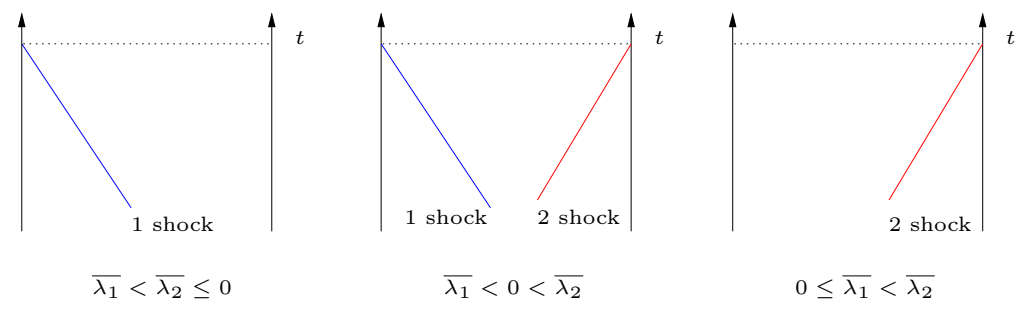

Figure 10: Strong shocks on the sides

That is, according to the signs of the characteristic speeds at $\bar{u}_{1}$, we make one or two strong shocks enter the domain, that is, we complete the final state on the left of 0 and on the right of 1 by states that naturally generate shocks (backward in time), as we did in the first step.

Also, an important property that we require from the shock(s) is to have characteristic speeds "under the shocks" bounded away from 0 . 
Initiating the backward front-tracking algorithm. Now we can begin to set up the backward fronttracking algorithm. Using the assumptions on $u_{1}$, we find particular piecewise constant approximations of $u_{1}$ : at each discontinuity point, we can "approximately" solve the backward Riemann problem using

- either "shock fans",

- either single rarefaction fronts with small amplitude, and with large distance between them,

- either the shocks that come from the boundary.

This is described in figure 11 .

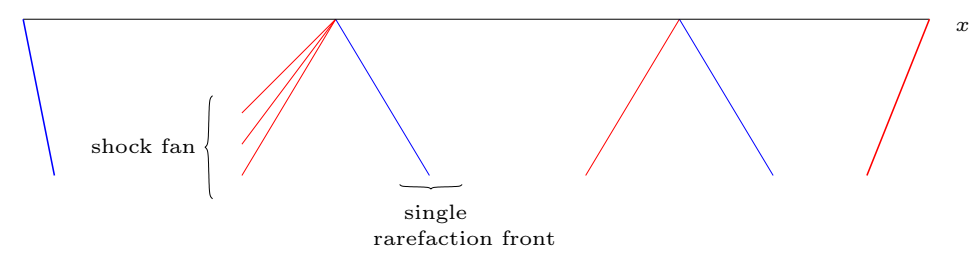

Figure 11: Beginning of the backward front-tracking approximation

Backward interactions. The next step in implementing the front-tracking algorithm backward in time is to solve backward interactions, that is, extend the approximation over interaction points (where two fronts meet). This is discussed according to the nature of the interaction.

- Interactions inside a family. We consider the case where two 1-fronts or two 2-fronts meet. Again, this depends on the nature of the incoming fronts.

- Shock/shock interactions: such interactions do not occur (as follows from Lax's inequalities).

- Rarefaction front/shock interactions: such interactions do not occur either (this follows also from Lax's inequalities, and from estimates on the sizes of the rarefaction fronts with respect to the size of the shocks).

- Rarefaction/rarefaction interactions: these interactions are likely, but must be avoided. Indeed, the only reasonable way to extend the approximation over such interaction points would be to "merge" these two fronts into a single one. But this would generate bigger rarefaction fronts, in such a way that, in the end, the extracted limit might not be entropic.

- Interactions for different families. Let us now consider the interaction point of a 1-front with a 2-front. The way we solve these interactions depends on the nature of the incoming fronts, that is, whether they are rarefaction fronts, (weak) shocks or strong shocks (successors of the fronts that originally come from 0 or 1$)$.

- Weak waves: if both waves are weak waves, one can "solve" the interactions, just as in Lax's Theorem. See figure 12 .

- Strong shock/weak shock interaction: again, one can extend the solution by a strong shock and a weak shock, satisfying Schochet's interaction estimates. See figure 13. 

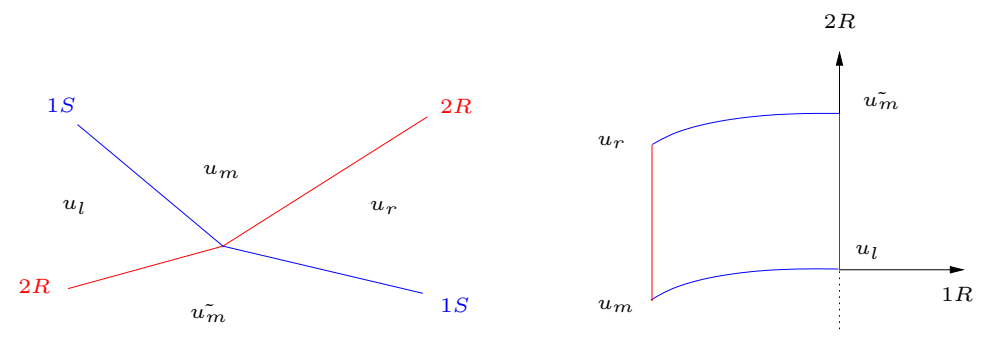

Figure 12: Weak-weak interactions.

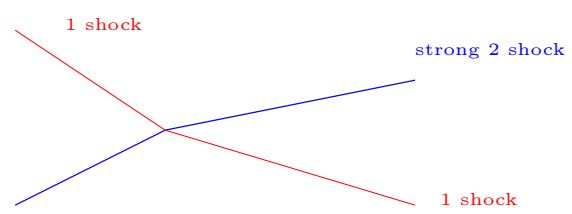

Figure 13: Strong shock-weak shock interactions.

- Strong shock/weak rarefaction interaction: we solve the backward interaction in terms of two incoming shocks of the same family (one strong, one weak). See figure 14. This is where we use the fact that we have some freedom in the backward Riemann problem. We could have extended such situation in terms of a strong shock and a rarefaction front, but this is an opportunity to get rid of a rarefaction front, which as we saw earlier (when discussing interactions inside a family) is favorable to our purpose.

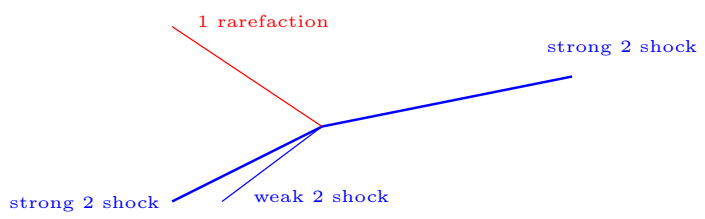

Figure 14: Strong shock-weak rarefaction front interactions.

To prove that the above algorithm is well-posed and yields an entropy solution to our problem, the main point is to estimate the distance between consecutive rarefaction fronts of the same family, since, as we saw earlier, we want to avoid the meeting of two rarefaction fronts of the same family. In fact, we prove that before possibly meeting, two such rarefaction fronts must

- either leave the domain,

- either be killed by the meeting of a strong shock of the opposite family.

This is done by using Glimm and Lax's estimates [18] on the spreading of rarefaction waves. This is indeed rather natural that their estimates on the spacing of characteristics (in the usual motion of the time) gives estimates on the focussing of rarefaction fronts backward in time. This is, of course, during that part of the proof that the form of the Oleinik-type conditions in Theorem 2 appear. This is illustrated in figure 15. 


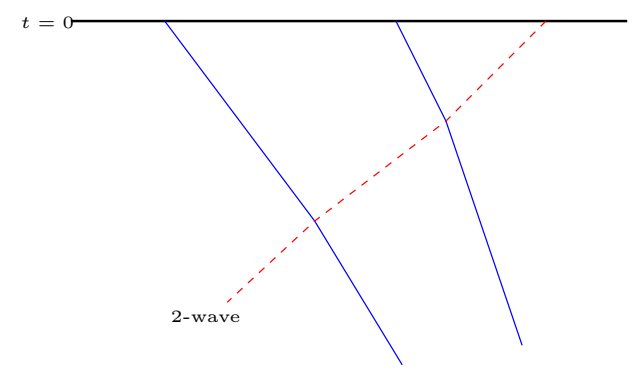

Figure 15: Focusing of rarefaction fronts.

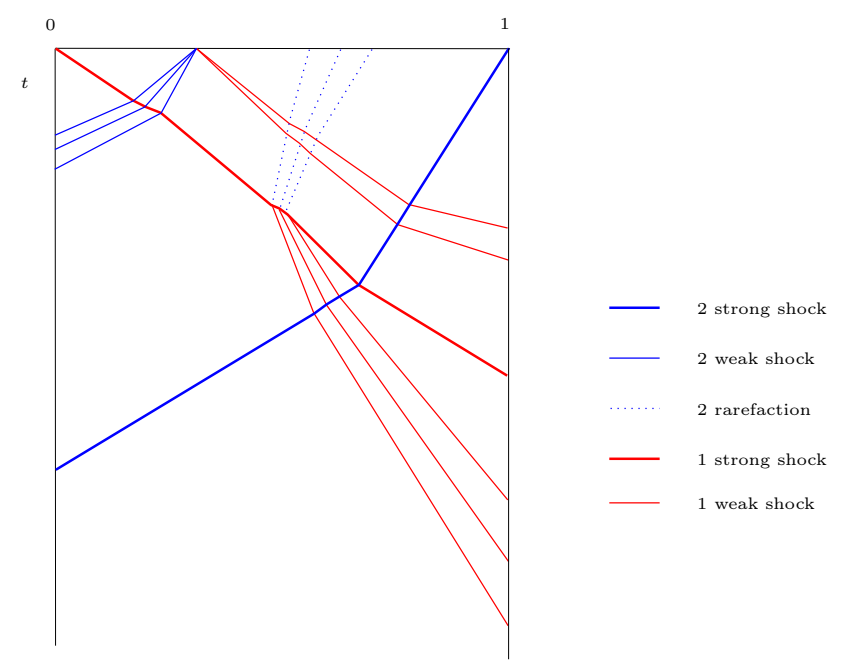

Figure 16: Backward front-tracking approximation

At the end, we get an approximation like is illustrated in Figure 16.

Then once we have proved that rarefaction fronts do not merge, the rest of the proof is very close to the usual one concerning the Cauchy problem.

\section{The Lagrangian case}

Let us now consider the case of the $p$-system (P). The above steps 2 and 3 are similar and even simpler because characteristic speeds are of fixed sign. The main difference in the Lagrangian case concerns the first step, that is, to get rid of the initial condition.

In fact, if we tried to use the previous strategy then this would fail. Indeed, if all we do is let a strong 2 -shock enter the domain, then it will certainly not make the first characteristic speed on the left of the strong shock be positive. What it will do is filter the 2 -waves for a short time, that is, there will be no 2 -fronts that directly emerge on the left of the 2-strong shock. But the 1-fronts cross it, and then their interactions yield 2-waves; so what we could think as a benefit of the 2-strong shock, vanishes shortly after that. This is illustrated in figure 17 .

However, we can see that, during that attempt, if there were only 1-rarefaction waves above the 2-strong shock, the problem would be solved. Indeed, two rarefaction fronts of the same family do not meet when 


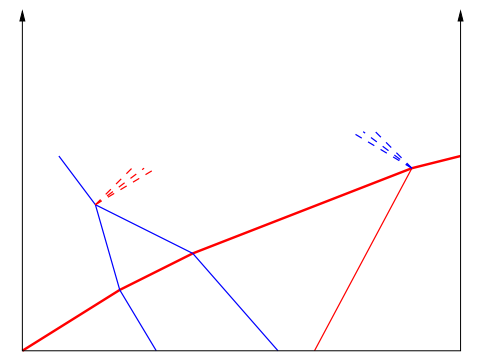

Figure 17: The filtering of 2-waves fails.

going forward in time. Hence, we would like to allow only 1-rarefaction fronts to cross the strong 2-shock, and find a way to discard the 1-shocks that emerge from the strong 2-shock.

There are two situations that can make a 1-shock enter the domain above the 2-strong shock:

- the meeting of the strong shock with a 1-shock,

- the meeting of the strong shock with a 2-rarefaction front.

This is described in figure 18 .
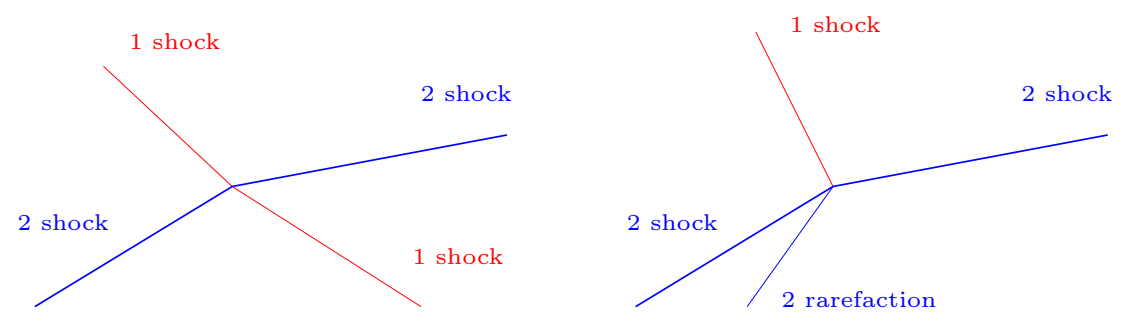

Figure 18: Situations which generate a 1-shock above the strong 2-shock

Now the main idea is that a 2-shock coming from the left and arriving exactly at the interaction point can kill the outgoing 1-shock. Hence, we prove that one can construct 2-shocks that kill the outgoing shock in the manner described in figure 19.
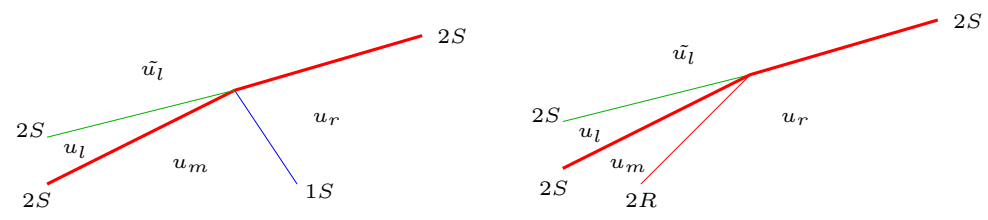

Figure 19: Additional 2-shocks

The previous statement is rather natural: it is known that for system (P) (as well as for (EI)), the meeting of two shocks of the same family generate a rarefaction wave in the other family. Hence gauging correctly the additional 2-shock on the left, one can hope to discard the 1-shock that would be generated by the interaction of the two fronts on the right only. 
Now, when we have proved that such additional 2-shocks exist, it remains to construct the approximation in which these 2 -shocks come at the right time and with the right strength. This is done in two steps.

First, we construct the solution under the 2-strong shock, taking the additional 2-shocks described above in to account: see figure 20

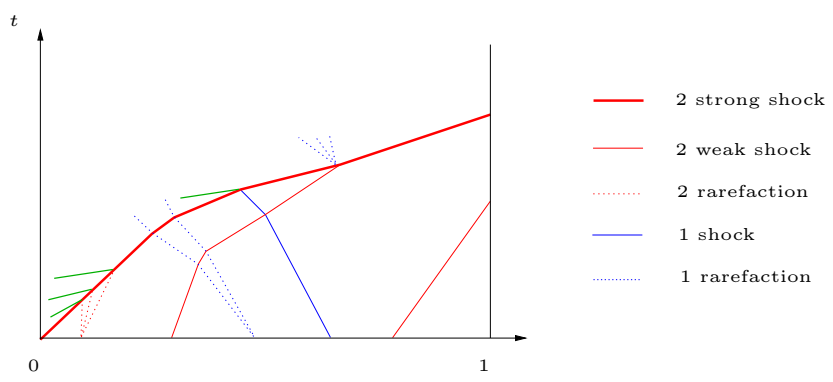

Figure 20: First step of the construction

This is done as in the usual front-tracking algorithm, except that we do not extend the approximation over the strong 2-shock (but we do remember the state on the left of the 2-strong shock).

Then, during the second step, it remains to construct the approximations beyond the strong 2-shock, that is, we have to extend the fronts that emerge from the strong 2-shock. These are of two types:

- the 1-rarefaction fronts that travel forward in time,

- the 2-shocks (that we have added) that travel backward in time.

The main idea to construct this approximation is to use $1-x$ as the time variable. Hence we are led to a problem in a varying domain, with boundary conditions on the "moving boundary" given by the strong 2 -shock constructed in the first step. This is illustrated in figure 21.

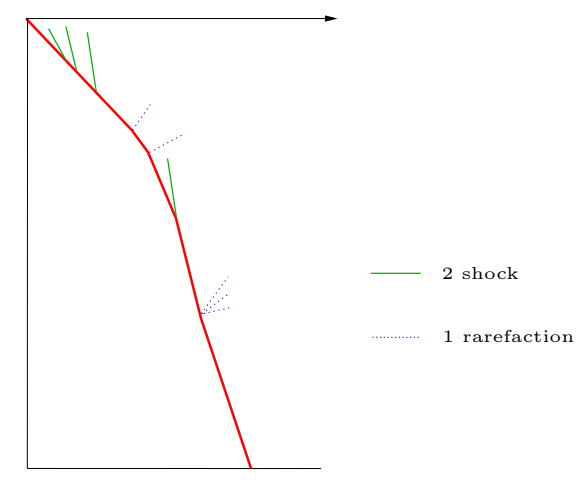

Figure 21: A problem in a varying domain.

The "germs" of fronts outgoing from the strong 2-shock were determined in the first step. What we have to do to extend this approximation is to solve the "interactions".

When studying these interactions, we see that there are only two possible types: 
- Interactions inside a family. There are no interactions of fronts of the same family because:

- rarefaction fronts go forward in time,

- shocks go backward in time,

and such fronts do not meet. Note in particular the important fact, that the extensions of the 2-shocks that we draw, do not get into the strong 2 -shock.

- Interactions of opposite family. These interactions can happen. It turns out that these "side" interactions of fronts of different families can be solved exactly as in Lax's Theorem. This is described in figure 22 .

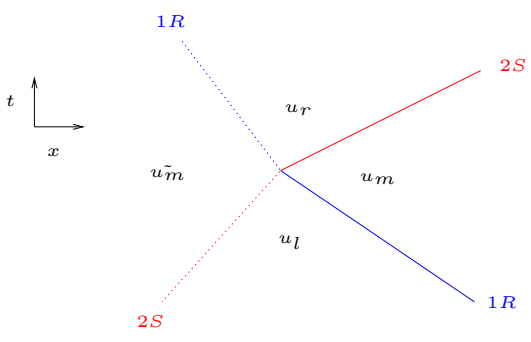

Figure 22: Side interactions.

Finally using the above algorithm we get an approximation like in figure 23.

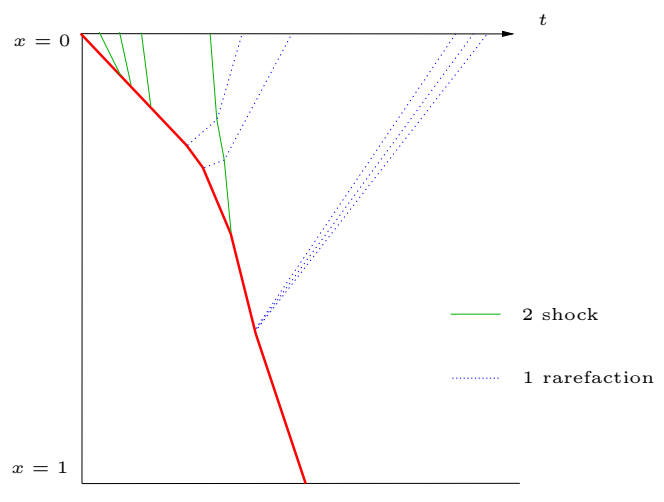

Figure 23: Second step.

In this approximation, the fronts above the strong 2 -shock are rarefaction fronts. Since they do not interact with each other and since there speed is not null (see the characteristic speeds of $(\mathrm{P})$ ), they leave the domain, which yields a constant state.

The rest of the proof is devoted to establish uniform $B V$ bounds on the approximation that we have constructed. This is done by introducing Glimm functionals on curves that are adapted to the construction, and the rest of the proof follows the classical lines of the proof concerning the Cauchy problem. 


\section{References}

[1] Alber H.-D., Local existence of weak solutions to the quasilinear wave equation for large initial values. Math. Z. 190 (1985), no. 2, pp. 249-276.

[2] Ancona F., Bressan A., Coclite G.M., Some results on the boundary control of systems of conservation laws. Hyperbolic problems: theory, numerics, applications, pp. 255-264, Springer, Berlin, 2003.

[3] Ancona F., Coclite G.M., On the attainable set for Temple class systems with boundary controls, Preprint SISSA (2002).

[4] Ancona F., Marson A., On the attainable set for scalar nonlinear conservation laws with boundary control. SIAM J. Control Optim. 36 (1998), no. 1, pp. 290-312.

[5] Beauchard K., Local controllability of a 1-D Schrödinger equation, J. Math. Pures Appl. 84 (2005), no. 7, pp. 851-956.

[6] Bressan A., Hyperbolic systems of conservation laws, the one-dimensional problem, Oxford Lecture Series in Mathematics and its Applications 20, 2000.

[7] Bressan A., Coclite G.M., On the boundary control of systems of conservation laws. SIAM J. Control Optim. 41 (2002), no. 2, 607-622

[8] Corli A., Sablé-Tougeron M., Perturbations of bounded variation of a strong shock wave. J. Differential Equations 138 (1997), no. 2, pp. 195-228.

[9] Coron J.-M., Global Asymptotic Stabilization for controllable systems without drift, Math. Control Signal Systems, 5, 1992, pp. 295-312.

[10] Coron J.-M., On the controllability of 2-D incompressible perfect fluids, J. Math. Pures Appl., 75 (1996), no. 2 , pp. $155-188$.

[11] Coron J.-M. Local controllability of a 1-D tank containing a fluid modeled by the shallow water equations. A tribute to J. L. Lions. ESAIM Control Optim. Calc. Var. 8 (2002), pp. 513-554.

[12] Dafermos C. M., Polygonal approximations of solutions of the initial value problem for a conservation law. J. Math. Anal. Appl. 38 (1972), pp. 33-41.

[13] Di Perna R. J., Global solutions to a class of nonlinear hyperbolic systems of equations. Comm. Pure Appl. Math. 26 (1973), pp. 1-28.

[14] Dubois F., LeFloch P.G., Boundary conditions for nonlinear hyperbolic systems of conservation laws. J. Differential Equations 71 (1988), no. 1, pp. 93-122.

[15] Glass O., Exact boundary controllability of 3-D Euler equation, ESAIM Control Optim. Calc. Var. 5 (2000) pp. 1-44.

[16] Glass O., On the controllability of the Vlasov-Poisson system. J. Differential Equations 195 (2003), no. 2, pp. 332-379.

[17] Glimm J., Solutions in the large for nonlinear hyperbolic systems of equations. Comm. Pure Appl. Math. 18 (1965), pp. 697-715.

[18] Glimm J., Lax, P. D., Decay of solutions of systems of nonlinear hyperbolic conservation laws. Memoirs of the A.M.S. 101 (1970).

[19] Horsin T., On the controllability of the Burgers equation. ESAIM: Control Opt. Calc. Var. 3 (1998), pp. 83-95. 
[20] Lax P. D., Hyperbolic Systems of Conservation Laws. Comm. Pure Appl. Math. 10 (1957), pp. 537-566.

[21] Lax P. D., Hyperbolic systems of conservation laws and the mathematical theory of shock waves. CBMS Regional Conference Series in Applied Mathematics, No. 11. SIAM, Philadelphia, 1973.

[22] Li T.-T.; Rao B.-P., Exact boundary controllability for quasi-linear hyperbolic systems. SIAM J. Control Optim. 41 (2003), no. 6, pp. 1748-1755.

[23] Lions P.-L., Perthame B., Souganidis P. E., Existence and stability of entropy solutions for the hyperbolic systems of isentropic gas dynamics in Eulerian and Lagrangian coordinates. Comm. Pure Appl. Math. 49 (1996), no. 6, pp. 599-638.

[24] Majda A., Compressible Fluid Flow and Systems of Conservation Laws in Several Space Variables, Springer-Verlag, New-York, 1984.

[25] Risebro, N. H., A front-tracking alternative to the random choice method. Proc. Amer. Math. Soc. 117 (1993), no. 4, pp. 1125-1139.

[26] Sablé-Tougeron M., Stabilité de la structure d'une solution de Riemann à deux grands chocs. Ann. Univ. Ferrara Sez. VII (N.S.) 44 (1998), pp. 129-172.

[27] Schochet S., Sufficient conditions for local existence via Glimm's scheme for large BV data. J. Differential Equations 89 (1991), no. 2, 317-354.

[28] Wagner D., Equivalence of the Euler and Lagrangian equations of gas dynamics for weak solutions. J. Differential Equations 68 (1987), no. 1, pp. 118-136. 